\title{
Rational Optimization using Sum-of-Squares Techniques
}

\author{
Javad Lavaei, Somayeh Sojoudi and Amir G. Aghdam
}

\begin{abstract}
Motivated by many control applications, this paper deals with the global solutions of unconstrained optimization problems. First, a simple SOS method is presented to find the infimum of a polynomial, which can be handled efficiently using the relevant software tools. The main idea of this method is to introduce a perturbation variable whose approaching to zero results in a solution with any arbitrary precision. The proposed technique is then extended to the case of rational functions. The primary advantages of this approach over the existing ones are its simplicity and capability of treating problems for which the existing methods are not efficient, as demonstrated in three numerical examples.
\end{abstract}

\section{INTRODUCTION}

In the past few years, there has been a particular interest towards applications of positive polynomials and sum-ofsquares (SOS) in various control problems. A SOS technique, for instance, provides the possibility of checking the stability of a wide range of nonlinear systems, for which the classical approaches often fail [1]. Furthermore, verifying the robust stability of a (polynomially) uncertain system is of special importance, which can be efficiently treated using SOS [2], [3], [4]. Some other key problems addressed using similar tools are the fixed-order $H_{\infty}$ controller design, pole placement by means of static output feedback and simultaneous stabilization. The recent book [5] has collected several new papers addressing the aforementioned problems, and manifestly casts light on the importance of SOS, LMI and positive polynomials in control. It is shown in [6] that several robust feedback design problems such as multiple performance specifications and robust performance can be formulated as the verification of the positivity of a set of polynomials over a specific region. Interestingly, most of the techniques proposed on these problems have been implemented in some software toolboxes such as YALMIP, SOSTOOLS, GloptiPoly [1], [7], [8].

It is well-known that the problems for which SOS techniques may be helpful are closely related to optimization. More precisely, many of the practical problems in control can be addressed in the context of either unconstrained or constrained optimization. An important class of optimization problems is the one involving minimization of a rational

This work has been supported by the Natural Sciences and Engineering Research Council of Canada under grant RGPIN-262127-07.

Javad Lavaei is with the Department of Control and Dynamical Systems, California Institute of Technology, Pasadena, USA (email: lavaei@cds.caltech.edu).

Somayeh Sojoudi is with the Department of Electrical and Computer Engineering, Concordia University, Montreal, Canada (email: s_sojoud@ece.concordia.ca).

Amir G. Aghdam is with the Department of Electrical and Computer Engineering, Concordia University, Montreal, Canada (email: aghdam@ece.concordia.ca). function, which is sometimes subject to certain rational inequalities. Problems of this type arise in several practical applications, some of which are listed below:

- The problem of identifying the state-space model of a structural dynamical system satisfying some constraints can be translated to the problem of minimizing a rational function subject to some rational constraints [9]. The main challenge in this problem is how to find the global solution as opposed to a local one.

- In the constrained model predictive control, where it is desired to predict the controlled variables over a future horizon, the minimization of a polynomial subject to some polynomial constraints is to be carried out in order to treat the problem [10], [11].

- Certain robust control problems such as parametric stability margin computation, can be formulated as the verification of the positivity of a polynomial on a hyperrectangle, as pointed out in [12].

- The minimum norm problem, which is investigated in the literature intensively, turns out to be equivalent to finding the global optimum of a polynomially constrained optimization problem [13].

- Minimization of a rational function is inevitably required in the problem of optimal model reduction [14] (and in the special case of finite impulse response (FIR) digital systems, the problem reduces to the minimization of a polynomial).

- Some important applications of polynomially constrained optimizations in control are explored in [1]; e.g., robust stability analysis, simultaneous stabilization, minimum distance to a surface, pole assignment via static output feedback and nonlinear stability analysis.

The practical applications described above point to the viable role of the aforementioned optimization problem in the real-world systems.

This paper tackles the problem of global optimization by means of SOS tools. A method is first proposed to obtain the infimum of a polynomial via a new perturbation technique. This SOS approach requires that a hierarchy of semi-definite programming (SDP) problems be solved. More precisely, the perturbation variable is initialized based on the required accuracy for the infimum being obtained, and then a family of SDPs is solved accordingly. The proposed approach is then extended to the case of finding the infimum of a rational function. Various aspects of the underlying ideas are explored here. The main advantage of this work over the existing methods (which are able to compute the exact infimum rather than a lower bound on it) is its simplicity. 
The paper is organized as follows. First, the existing methods for solving a rational optimization problem are pointed out in Section II, and their advantages and disadvantages are discussed thoroughly. The problems of finding the infimums of polynomials and rational functions are then investigated in Section III and Section IV, respectively. Three illustrative numerical examples are presented in Section V. Finally, some concluding remarks are given in Section VI.

\section{PRELIMINARIES}

Consider a polynomial $f(\mathbf{x})$, where $\mathbf{x}=$ $\left[\begin{array}{llll}x_{1} & x_{2} & \cdots & x_{n}\end{array}\right]$, and denote its infimum with $\alpha_{*}$. In the recent years, the problem of finding $\alpha_{*}$ by means of semidefinite programming has been investigated in the literature intensively. These works will be surveyed below, and their properties will be discussed.

It is manifest that $\alpha_{*}$ is equal to the supremum of all values of $\alpha$ for which the polynomial $f(\mathbf{x})-\alpha$ is nonnegative. It is notable that this idea has first been used in [15] for the general case. The works [16] and [17] replace the condition of $f(\mathbf{x})-\alpha$ being nonnegative with a stronger condition of being SOS in order to further simplify the underlying problem. This leads to a lower bound on $\alpha_{*}$, which can be a wretchedly conservative solution. For instance, consider the polynomial:

$$
x_{1}^{4} x_{1}^{2}+x_{1}^{2} x_{2}^{4}+1-3 x_{1}^{2} x_{2}^{2}
$$

Using the work [17], the lower bound $-\infty$ is obtained for the infimum of this polynomial, whereas the infimum is equal to 0 . The weakness of this method can be justified by the result obtained in [18], which states that the ratio of the volume of the nonnegative non-SOS homogeneous polynomials of degree $2 d$ to the volume of the SOS homogeneous polynomials with the same degree grows rapidly towards infinity, as $n$ goes to infinity, for any fixed integer $d \geq 2$.

Assume that the infimum of $f(\mathbf{x})$ is attainable and is known to lie inside a ball of radius $R$ centered at the origin. The work [16] employes a famous result on positivity in the unit ball (e.g. see [19]) to assert that $\alpha_{*}$ is equal to the supremum of all values of $\alpha$ for which there exist two SOS polynomials $\phi_{1}(\mathbf{x})$ and $\phi_{2}(\mathbf{x})$ with the following property:

$$
f(\mathbf{x})-\alpha=\left(R^{2}-\mathbf{x x}^{T}\right) \phi_{1}(\mathbf{x})+\phi_{2}(\mathbf{x})
$$

Although this SOS formulation seems appealing from the mathematical perspective, it is useful only when the following questions can be addressed according to the available $a$ priori information:

- Does $f(\mathbf{x})$ have a finite infimum?

- Can $f(\mathbf{x})$ attain its infimum, if it exists (i.e., does there exist a finite point corresponding to that infimum)?

- If the infimum is attainable, how can the radius $R$ be determined?

The open questions given above (and in particular the last one) make this approach ad-hoc in general. It is worth mentioning that one may speculate that considering a very large value for $R$ alleviates the issue to some degree; however, this can potentially make the corresponding SOS problem run into numerical difficulties (see Example 4 of [20] and the discussion given therein). In other words, there is a trade-off between the magnitude of $R$ and the accuracy of the solution. Note that this technique is also utilized in [21].

The method proposed in [22] attempts to bridge the gap between SOS polynomials and nonnegative polynomials, which may potentially resolve the deficiency of the works [16] and [17] to some extent. Consider a nonnegative polynomial $p(\mathbf{x})$. It is shown in [22] that for any $\varepsilon>0$, there exists an integer $r$ depending on $p(\mathbf{x})$ and $\varepsilon$ such that the polynomial:

$$
p(\mathbf{x})+\varepsilon \sum_{i=1}^{r} \sum_{j=1}^{n} \frac{x_{j}^{2 i}}{i !}
$$

is SOS. This elegant result incorporates the nonnegative polynomials into the SOS ones.

The work [23] deals with the problem of minimizing a polynomial $f(\mathbf{x})$. For any positive number $\varepsilon$, it defines the polynomial:

$$
f_{\varepsilon}(\mathbf{x}):=f(\mathbf{x})+\varepsilon \sum_{i=1}^{n} x_{i}^{2 \sigma+2}
$$

where $2 \sigma$ denotes the degree of $f(\mathbf{x})$. The method proposed in [23] asserts the following advantages of $f_{\varepsilon}(\mathbf{x})$ :

- The infimum of $f_{\varepsilon}(\mathbf{x})$ approaches that of $f(\mathbf{x})$, as $\varepsilon$ goes to zero.

- Although $f(\mathbf{x})$ may not attain its infimum, $f_{\varepsilon}(\mathbf{x})$ always attains the corresponding infimum.

Subsequently, the problem of finding the minimum of $f_{\varepsilon}(\mathbf{x})$ is converted to a generalized eigenvalue problem. The main drawback associated with this approach is that the entries of a certain matrix used in the algorithm proposed therein are expressed in terms of $\frac{1}{\varepsilon}$, which prevents $\varepsilon$ from going to zero (in order to obtain a precise solution).

The results of [23] have been further developed in [20]. It is shown that the infimum of the perturbed $f_{\varepsilon}(\mathbf{x})$ given by (4) is inside a ball. The radius of this ball is also obtained in [20]. The ball technique discussed earlier is exploited subsequently to find the infimum of $f(\mathbf{x})$. Nonetheless, this approach has some important shortcomings. First of all, the radius of said ball is proportional to $\frac{n^{\sigma}}{\varepsilon}$, which is usually very large and causes numerical difficulties. Moreover, some of the values used in the corresponding formulation are expressed in terms of $\frac{1}{\varepsilon}$. These facts result in an ill-conditioned optimization problem, for which $\varepsilon$ should be considered neither small (due to the mentioned difficulties) nor large (due to the required accuracy). However, unlike the other existing methods which seek a lower bound for $\alpha_{*}$, the work [20] presents an upper bound for it.

It is shown in [24] that the infimum of $f(\mathbf{x})$ is equal to the supremum of all values of $\alpha$ for which there exist a SOS polynomial $\phi_{0}(\mathbf{x})$ and polynomials $\phi_{1}(\mathbf{x}), \ldots, \phi_{n}(\mathbf{x})$ such that:

$$
f(\mathbf{x})-\alpha=\phi_{0}(\mathbf{x})+\phi_{1}(\mathbf{x}) \frac{\partial f(\mathbf{x})}{\partial x_{1}}+\cdots+\phi_{n}(\mathbf{x}) \frac{\partial f(\mathbf{x})}{\partial x_{n}}
$$


This method is contingent upon the assumption that $f(\mathbf{x})$ attains its infimum, which is by no means an easy-tocheck assumption. Moreover, the number of polynomials involved in the proposed SOS formulation becomes large, as $n$ increases. This yields a high-dimension SOS problem in general, and from this point of view, it differs from the works using the big ball technique (because those works seek merely two polynomials).

The work [25] tackles the global optimization of a polynomial $f(\mathbf{x})$. One of the requirements of the approach in [25] is that $f(\mathbf{x})$ should be bounded from below. This helps improve the effectiveness of this approach, distinguishing it from the one in [24] which is unable to deal with the polynomials whose infimums are unattainable. The work [25] introduces the notion of a principal gradient tentacle and uses it instead of the gradient variety notion in [24]. The gradient tentacle of the polynomial $f(\mathbf{x})$ is defined as:

$$
S(\nabla \mathbf{f}(\mathbf{x}))=\{\mathbf{x}:\|\nabla f(\mathbf{x})\|\|\mathbf{x}\| \leq 1\}
$$

where:

$$
\|\nabla f(\mathbf{x})\|^{2}=\left(\frac{\partial f(\mathbf{x})}{\partial x_{1}}\right)^{2}+\cdots+\left(\frac{\partial f(\mathbf{x})}{\partial x_{n}}\right)^{2}
$$

It is then stated that if $f(\mathbf{x})$ has isolated singularities only at infinity, or alternatively if $S(\nabla f(\mathbf{x}))$ is compact, then $\alpha_{*}$ is equal to the supremum of all values of $\alpha$ for which there exist two SOS polynomials $\phi_{1}(\mathbf{x})$ and $\phi_{2}(\mathbf{x})$ such that:

$$
f(\mathbf{x})-\alpha=\phi_{1}(\mathbf{x})+\left(1-\|\nabla f(\mathbf{x})\|^{2}\|\mathbf{x}\|^{2}\right) \phi_{2}(\mathbf{x})
$$

If the infimum of $f(\mathbf{x})$ does not exist, this method fails to observe it, and hence may lead to a wrong solution. Furthermore, in the case when the infimum is not attainable, this method can be inefficient in terms of time (as pointed out in [25]). Comparing the approach in [25] with other existing methods, one can easily infer that the term $\|\nabla f(\mathbf{x})\|^{2}\|\mathbf{x}\|^{2}$ will increase the numerical complexity of the problem noticeably (because of its degree).

The drawbacks of the aforementioned methods support the importance of developing more effective procedures for this type of global optimization problems. The following notations will prove to be convenient in the presentation of the main results.

Notation 1:

- Denote the unit sphere in $\Re^{k}$ with $\mathcal{S}^{k}$, for any natural number $k$.

- Denote the set of all SOS polynomials with $\Sigma^{2}$.

- Denote the set of all real-valued polynomials with $\Re[\mathbf{X}]$.

- Denote the set of all real-valued polynomials of degree $k$ with $\Re_{k}[\mathbf{X}]$, for any nonnegative integer $k$.

Notation 2: For any rational function $\frac{p_{1}(\mathbf{x})}{p_{2}(\mathbf{x})}$, the domain of definition of this function can normally be considered as one of the following:

i) every $\mathbf{x}$ for which $p_{2}(\mathbf{x})$ is nonzero;

ii) every $\mathbf{x}$ for which the limit of $\frac{p_{1}(\mathbf{x})}{p_{2}(\mathbf{x})}$ exists;

iii) every $\mathbf{x}$ for which $q_{2}(\mathbf{x})$ is nonzero, where $q_{2}(\mathbf{x})$ is obtained from $p_{2}(\mathbf{x})$ after eliminating its common divisors with $p_{1}(\mathbf{x})$.
Throughout this paper, the domain of definition of a rational function refers to the domain (iii) given above.

Notation 3: Consider a polynomial $p(\mathbf{x})$. For each term of this polynomial, namely $\gamma_{g} x_{1}^{g_{1}} x_{2}^{g_{2}} \ldots x_{n}^{g_{n}}$, compute the quantity $\frac{g_{1} ! g_{2} ! \ldots g_{n} !}{\left(g_{1}+g_{2}+\cdots+g_{n}\right) !}\left|\gamma_{g}\right|$. Let $\|p(\mathbf{x})\|$ denote the maximum value of these quantities corresponding to all different terms (see [28]).

The following lemma is extracted from [19], which can be envisaged as a particular case of Putinar's theorem [26].

Lemma 1: Given a polynomial $p(\boldsymbol{\omega}): \Re^{\lambda} \rightarrow \Re$, there exist polynomials $\phi_{1}(\boldsymbol{\omega}) \in \Re[\mathbf{X}]$ and $\phi_{2}(\boldsymbol{\omega}) \in \Sigma^{2}$ such that:

$$
p(\boldsymbol{\omega})=\left(1-\boldsymbol{\omega} \boldsymbol{\omega}^{T}\right) \phi_{1}(\boldsymbol{\omega})+\phi_{2}(\boldsymbol{\omega})
$$

if $p(\boldsymbol{\omega})$ is positive on the $\lambda$-dimensional unit sphere. Moreover, when $p(\boldsymbol{\omega})$ is negative at some point on the unit sphere, there do not exist any $\phi_{1}(\boldsymbol{\omega}) \in \Re[\mathbf{X}]$ and $\phi_{2}(\boldsymbol{\omega}) \in \Sigma^{2}$ satisfying the equation (9).

\section{THE INFIMUM OF A POLYNOMIAL}

Consider a polynomial $f(\mathbf{x})$ of degree $2 \sigma$ and denote its infimum with $\alpha_{*}$. It is evident that $\alpha_{*}$ can be obtained from the following relation:

$$
\alpha_{*}=\sup \left\{\alpha \mid f(\mathbf{x})-\alpha \geq 0, \forall \mathbf{x} \in \Re^{n}\right\}
$$

Let $\mu$ represent a slack variable. Rewrite the function $f\left(\frac{\mathbf{x}}{\mu}\right)$ as $\mu^{-2 \sigma} \bar{f}(\mathbf{x}, \mu)$, where $\bar{f}(\mathbf{x}, \mu)$ is a homogeneous polynomial.

Definition 1: For any nonnegative integer $k$ and any $\varepsilon \geq$ 0 , define $\alpha_{\varepsilon}(k)$ as the supremum of all values of $\alpha$ for which there exist polynomials $\phi_{1}^{\varepsilon}(\mathbf{x}, \mu) \in \Re_{k}[\mathbf{X}]$ and $\phi_{2}^{\varepsilon}(\mathbf{x}, \mu) \in$ $\Sigma^{2}$ such that:

$$
\begin{aligned}
\bar{f}(\mathbf{x}, \mu)+\varepsilon-\alpha\left(\mu^{2 \sigma}+\varepsilon^{2}\right) & =\left(1-\mathbf{x x}^{T}-\mu^{2}\right) \phi_{1}^{\varepsilon}(\mathbf{x}, \mu) \\
& +\phi_{2}^{\varepsilon}(\mathbf{x}, \mu)
\end{aligned}
$$

Note that $\alpha_{\varepsilon}(k)$ can be equal to $-\infty$ for some $k$, in which case the relevant polynomials do not exist. The sequence $\left\{\alpha_{\varepsilon}(k)\right\}_{k=0}^{\infty}$ is obviously monotone non-decreasing, and hence one can define the following limit:

$$
\alpha_{\varepsilon}:=\lim _{k \rightarrow+\infty} \alpha_{\varepsilon}(k)
$$

Remark 1: It is interesting to note that as substantiated in Proposition 2 of [29], the quantity $\alpha_{\varepsilon}$ is alternatively equal to the supremum of all values of $\alpha$ for which there exists some integer $r \geq 0$ such that:

$$
\left(\bar{f}(\mathbf{x}, \mu)+\varepsilon-\alpha\left(\mu^{2 \sigma}+\varepsilon^{2}\right)\right)\left(\mathbf{x x}^{T}+\mu^{2}\right)^{r} \in \Sigma^{2}
$$

In other words, the Putinar representation (see [26]) and the Pólya representation (given in (13)) can both be used to find $\alpha_{\varepsilon}$.

Theorem 1: For any $\varepsilon>0$, the quantity $\alpha_{\varepsilon}$ is equal to the minimum of the rational function $\frac{\bar{f}(\mathbf{x}, \mu)+\varepsilon}{\mu^{2 \sigma}+\varepsilon^{2}}$ on the unit sphere $\mathcal{S}^{n+1}$. Furthermore, $\alpha_{0}$ (i.e., $\alpha_{\varepsilon}$ at $\varepsilon=0$ ) is a lower bound on the infimum of the rational function $\frac{\bar{f}(\mathbf{x}, \mu)}{\mu^{2 \sigma}}$ on the sphere $\mathcal{S}^{n+1}$. 
Proof: For any $\varepsilon \geq 0$, denote the infimum of the rational function $\frac{\bar{f}(\mathbf{x}, \mu)+\varepsilon}{\mu^{2 \sigma}+\varepsilon^{2}}$ on the sphere $S^{n+1}$ with $\hat{\alpha}_{\varepsilon}$. One can write:

$$
\begin{aligned}
\hat{\alpha}_{\varepsilon}=\sup \left\{\alpha \mid \bar{f}(\mathbf{x}, \mu)+\varepsilon-\alpha\left(\mu^{2 \sigma}+\varepsilon^{2}\right)\right. & \geq 0, \\
\forall(\mathbf{x}, \mu) & \left.\in \mathcal{S}^{n+1}\right\}
\end{aligned}
$$

Assume for now that $\varepsilon$ is strictly positive. Since $\mu^{2 \sigma}+\varepsilon^{2}$ is always strictly positive in this case, Lemma 1 in [21] along with the above equation yields that:

$$
\begin{array}{r}
\hat{\alpha}_{\varepsilon}=\sup \left\{\alpha \mid \bar{f}(\mathbf{x}, \mu)+\varepsilon-\alpha\left(\mu^{2 \sigma}+\varepsilon^{2}\right)>0,\right. \\
\left.\forall(\mathbf{x}, \mu) \in \mathcal{S}^{n+1}\right\}
\end{array}
$$

Now, it follows from [19] that $\hat{\alpha}_{\varepsilon}$ is identical to the supremum of all values of $\alpha$ for which there exist polynomials $\phi_{1}^{\varepsilon}(\mathbf{x}, \mu) \in \Re[\mathbf{X}]$ and $\phi_{2}^{\varepsilon}(\mathbf{x}, \mu) \in \Sigma^{2}$ satisfying the equation (11). This means that $\hat{\alpha}_{\varepsilon}$ and $\alpha_{\varepsilon}$ are identical for any strictly positive $\varepsilon$. However, since $\mu^{2 \sigma}+\varepsilon^{2}$ is not always strictly positive for $\varepsilon=0$, the results of [19] cannot be employed for $\varepsilon=0$. In this case, it can be easily shown that $\alpha_{0}$ is not necessarily equal to the infimum of the rational function $\frac{\bar{f}(\mathbf{x}, \mu)}{\mu^{2 \sigma}}$ on the sphere $\mathcal{S}^{n+1}$, and is indeed a lower bound on it.

Corollary 1: The number $\alpha_{0}$ is always less than or equal to the infimum $\alpha_{*}$.

Proof: In light of Theorem 1, $\alpha_{0}$ is a lower bound on the infimum of $\frac{\bar{f}(\mathbf{x}, \mu)}{\mu^{2 \sigma}}$ over $\mathcal{S}^{n+1}$. The proof follows immediately from the fact that the infimum of $f(\mathbf{x})$ in $\Re^{n}$ is the same as that of $\frac{\bar{f}(\mathbf{x}, \mu)}{\mu^{2 \sigma}}$ on $\mathcal{S}^{n+1}$.

Corollary 1 provides a lower bound on the infimum of the polynomial $f(\mathbf{x})$. Since the difference between $\alpha_{0}$ and $\alpha_{*}$ normally cannot be measured, it is not clear how tight the lower bound $\alpha_{0}$ is. To remedy this shortcoming, it will be shown in the sequel how arbitrarily tight bounds on the infimum $\alpha_{*}$ can be obtained.

Theorem 2: For any $\varepsilon$ satisfying the relation:

$$
0<\varepsilon<\max \left(\frac{1}{f(0)},-\operatorname{sgn}(f(0)) \times \infty\right)
$$

the inequality $\alpha_{*}<\alpha_{\varepsilon}$ holds, where $\operatorname{sgn}(\cdot)$ represents the sign function (note that if $f(0)$ is zero, then the inequality (16) is identical to $0<\varepsilon<\infty$ ).

Proof: It follows from the inequality (16) that $1-f(0) \varepsilon$ is greater than zero. Now, let $\beta$ denote an arbitrary number satisfying the relation:

$$
0<\beta<\frac{\varepsilon-f(0) \varepsilon^{2}}{1+\varepsilon^{2}}
$$

This along with the relation $\alpha_{*} \leq f(0)$ results in:

$$
\varepsilon-\alpha_{*} \varepsilon^{2}-\beta-\beta \varepsilon^{2}>0
$$

Hence, for any $(\mathbf{x}, \mu) \in \mathcal{S}^{n+1}$, one can write:

$$
\varepsilon-\alpha_{*} \varepsilon^{2}-\beta \mu^{2 \sigma}-\beta \varepsilon^{2}>0
$$

and subsequently:

$$
\bar{f}(\mathbf{x}, \mu)+\varepsilon-\left(\alpha_{*}+\beta\right)\left(\mu^{2 \sigma}+\varepsilon^{2}\right)>\bar{f}(\mathbf{x}, \mu)-\alpha_{*} \mu^{2 \sigma}
$$

for all $(\mathbf{x}, \mu) \in \mathcal{S}^{n+1}$. On the other hand, as a direct property of $\alpha_{*}$, the function $\bar{f}(\mathbf{x}, \mu)-\alpha_{*} \mu^{2 \sigma}$ is nonnegative on the unit sphere. Therefore:

$$
\bar{f}(\mathbf{x}, \mu)+\varepsilon-\left(\alpha_{*}+\beta\right)\left(\mu^{2 \sigma}+\varepsilon^{2}\right)>0, \quad \forall(\mathbf{x}, \mu) \in \mathcal{S}^{n+1}
$$

Now, it can be concluded from Lemma 1 that there exist two polynomials $\phi_{1}^{\varepsilon}(\mathbf{x}, \mu) \in \Re[\mathbf{X}]$ and $\phi_{2}^{\varepsilon}(\mathbf{x}, \mu) \in \Sigma^{2}$ such that:

$$
\begin{aligned}
\bar{f}(\mathbf{x}, \mu)+\varepsilon-\left(\alpha_{*}+\beta\right)\left(\mu^{2 \sigma}+\varepsilon^{2}\right) & =\left(1-\mathbf{x x}^{T}-\mu^{2}\right) \\
& \times \phi_{1}^{\varepsilon}(\mathbf{x}, \mu)+\phi_{2}^{\varepsilon}(\mathbf{x}, \mu)
\end{aligned}
$$

One can deduce directly from the above equation and the definition of $\alpha_{\varepsilon}$ that:

$$
\alpha_{\varepsilon} \geq \alpha_{*}+\beta
$$

The proof follows immediately on noting that $\beta$ is a strictly positive number.

Remark 2: Assume that $\varepsilon$ is chosen so that the inequality (16) is satisfied. It can be easily derived from the proof of Theorem 2 that $\alpha_{\varepsilon}$ is greater than $\alpha_{*}$ by at least $\frac{\varepsilon-f(0) \varepsilon^{2}}{1+\varepsilon^{2}}$. However, as $\varepsilon$ goes to zero, this difference vanishes.

Theorem 3: The function $\alpha_{\varepsilon}$ is monotone increasing (with respect to the variable $\varepsilon$ ) over the interval $\left(0,-f(0)+\sqrt{f(0)^{2}+1}\right)$.

Proof: Consider two real numbers $\varepsilon_{1}$ and $\varepsilon_{2}$ belonging to the open interval $\left(0,-f(0)+\sqrt{f(0)^{2}+1}\right)$, and assume with no loss of generality that $\varepsilon_{2}<\varepsilon_{1}$. Now, it suffices to prove that $\alpha_{\varepsilon_{2}}<\alpha_{\varepsilon_{1}}$. To this end, it is straightforward to show that:

$$
\varepsilon_{1}^{2}+2 f(0) \varepsilon_{1}-1<0
$$

Using this inequality and on employing Theorem 1, it is a matter of computation to substantiate that:

$$
\begin{aligned}
\alpha_{\varepsilon_{1}}=\min _{(\mathbf{x}, \mu) \in \mathcal{S}^{n+1}} \frac{\bar{f}(\mathbf{x}, \mu)+\varepsilon_{1}}{\mu^{2 \sigma}+\varepsilon_{1}^{2}} & \leq\left.\frac{\bar{f}(\mathbf{x}, \mu)+\varepsilon_{1}}{\mu^{2 \sigma}+\varepsilon_{1}^{2}}\right|_{\mathbf{x}=0, \mu=1} \\
& =\frac{f(0)+\varepsilon_{1}}{1+\varepsilon_{1}^{2}} \leq \frac{1}{2 \varepsilon_{1}}
\end{aligned}
$$

As a result:

$$
\alpha_{\varepsilon_{1}}<\frac{1}{\varepsilon_{1}+\varepsilon_{2}}
$$

This inequality points to the following relation:

$$
\begin{aligned}
\bar{f}\left(\overline{\mathbf{x}}_{\varepsilon_{1}}, \bar{\mu}_{\varepsilon_{1}}\right)+\varepsilon_{1}-\alpha_{\varepsilon_{1}}\left(\bar{\mu}_{\varepsilon_{1}}^{2 \sigma}+\varepsilon_{1}^{2}\right) & >\bar{f}\left(\overline{\mathbf{x}}_{\varepsilon_{1}}, \bar{\mu}_{\varepsilon_{1}}\right)+\varepsilon_{2} \\
& -\alpha_{\varepsilon_{1}}\left(\bar{\mu}_{\varepsilon_{1}}^{2 \sigma}+\varepsilon_{2}^{2}\right)
\end{aligned}
$$

where $\left(\overline{\mathbf{x}}_{\varepsilon_{1}}, \bar{\mu}_{\varepsilon_{1}}\right)$ denotes a minimizer of the function $\frac{\bar{f}(\mathbf{x}, \mu)+\varepsilon_{1}}{\mu^{2 \sigma}+\varepsilon_{1}^{2}}$ on the sphere $\mathcal{S}^{n+1}$. From Theorem 1, the left side of the above inequality is equal to 0 . This implies that:

$$
\bar{f}\left(\overline{\mathbf{x}}_{\varepsilon_{1}}, \bar{\mu}_{\varepsilon_{1}}\right)+\varepsilon_{2}-\alpha_{\varepsilon_{1}}\left(\bar{\mu}_{\varepsilon_{1}}^{2 \sigma}+\varepsilon_{2}^{2}\right)<0
$$

Thus:

$$
\alpha_{\varepsilon_{2}}=\min _{(\mathbf{x}, \mu) \in \mathcal{S}^{n+1}} \frac{\bar{f}(\mathbf{x}, \mu)+\varepsilon_{2}}{\mu^{2 \sigma}+\varepsilon_{2}^{2}} \leq \frac{\bar{f}\left(\overline{\mathbf{x}}_{\varepsilon_{1}}, \bar{\mu}_{\varepsilon_{1}}\right)+\varepsilon_{2}}{\bar{\mu}_{\varepsilon_{1}}^{2 \sigma}+\varepsilon_{2}^{2}}<\alpha_{\varepsilon_{1}}
$$

This completes the proof. 
Theorem 4: The infimum $\alpha_{*}$ can be obtained from the following relation:

$$
\alpha_{*}=\lim _{\varepsilon \rightarrow 0^{+}} \alpha_{\varepsilon}
$$

Proof: It can be inferred from Theorem 3 that the right limit of $\alpha_{\varepsilon}$ at $\varepsilon=0$ exists. On the other hand, it is known that:

$$
\alpha_{\varepsilon} \leq \frac{\bar{f}(\mathbf{x}, \mu)+\varepsilon}{\mu^{2 \sigma}+\varepsilon^{2}}, \quad \forall(\mathbf{x}, \mu) \in \mathcal{S}^{n+1}, \quad \forall \varepsilon>0
$$

Hence, taking the limit of both sides of the above inequality results in:

$$
\lim _{\varepsilon \rightarrow 0^{+}} \alpha_{\varepsilon} \leq \frac{\bar{f}(\mathbf{x}, \mu)}{\mu^{2 \sigma}}, \quad \forall(\mathbf{x}, \mu) \in \mathcal{S}^{n+1}, \quad \mu \neq 0
$$

Besides, the infimum of the function $\frac{\bar{f}(\mathbf{x}, \mu)}{\mu^{2 \sigma}}$ on the sphere $S^{n+1}$ is the same for both cases of the sphere including or excluding all the points with $\mu=0$. Applying this fact to the above inequality leads to:

$$
\lim _{\varepsilon \rightarrow 0^{+}} \alpha_{\varepsilon} \leq \alpha_{*}
$$

On the other hand, Theorem 2 states that:

$$
\alpha_{*}<\alpha_{\varepsilon}, \quad \forall \varepsilon \in\left(0, \max \left(\frac{1}{f(0)},-\operatorname{sgn}(f(0)) \times \infty\right)\right)
$$

The proof follows from the relations (33) and (34).

Theorem 5: Assume that the infimum of $f(\mathbf{x})$ is attainable. Let $R$ denote the radius of a ball centered at the origin, in which a minimizer of $f(\mathbf{x})$ is located. Moreover, denote a lower bound on $\alpha_{*}$ with $\alpha_{*}$. For any $\varepsilon$ satisfying all the relations:

$$
\begin{aligned}
& 0<\varepsilon<\frac{1}{4\left(1+R^{2}\right)^{2 \sigma}}, \\
& 0<\varepsilon<\max \left(\frac{1}{f(0)},-\operatorname{sgn}(f(0)) \times \infty\right), \\
& 0<\varepsilon<\max \left(\frac{1}{-\underline{\alpha_{*}}}, \operatorname{sgn}\left(\underline{\alpha_{*}}\right) \times \infty\right)
\end{aligned}
$$

the inequality given below holds:

$$
\alpha_{*}<\alpha_{\varepsilon}<\alpha_{*}+\sqrt{\varepsilon}
$$

(if $\alpha_{*}$ is equal to 0 , replace the inequality (35c) with $0<$ $\varepsilon<+\infty)$.

Proof: Consider a value for $\varepsilon$ pertaining to the open interval specified by the inequalities (35a), (35b) and (35c). By virtue of the fact that $\varepsilon$ satisfies the relation $(35 b)$, it results from Theorem 2 that $\alpha_{*}<\alpha_{\varepsilon}$. Now, it remains to prove the validity of the inequality $\alpha_{\varepsilon}<\alpha_{*}+\sqrt{\varepsilon}$. To accomplish this, denote with $\mathbf{x}_{*}$ a minimizer of the polynomial $f(\mathbf{x})$ lying in the ball of radius $R$ and centered at the origin. As pointed out in the proof of Corollary 1, the infimum of $\frac{\bar{f}(\mathbf{x}, \mu)}{\mu^{2 \sigma}}$ on the unit sphere $\mathcal{S}^{n+1}$ is equal to $\alpha_{*}$. It can be easily shown that this infimum corresponds to the $\operatorname{minimizer}\left(\overline{\mathbf{x}}_{*}, \bar{\mu}_{*}\right)$, where:

$$
\bar{\mu}_{*}=\frac{1}{\sqrt{1+\mathbf{x}_{*} \mathbf{x}_{*}^{T}}}, \quad \overline{\mathbf{x}}_{*}=\bar{\mu}_{*} \times \mathbf{x}_{*}
$$

(note that $\bar{\mu}_{*}^{2}+\overline{\mathbf{x}}_{*} \overline{\mathbf{x}}_{*}^{T}=1$ ). It is desired now to prove that:

$$
\bar{f}\left(\overline{\mathbf{x}}_{*}, \bar{\mu}_{*}\right)+\varepsilon-\left(\alpha_{*}+\sqrt{\varepsilon}\right)\left(\bar{\mu}_{*}^{2 \sigma}+\varepsilon^{2}\right)<0
$$

To this end, one can write:

$$
\bar{f}\left(\overline{\mathbf{x}}_{*}, \bar{\mu}_{*}\right)-\alpha_{*} \bar{\mu}_{*}^{2 \sigma}=0
$$

In addition, it can be easily verified that the inequality (35c) is tantamount to the following:

$$
\varepsilon \underline{\alpha_{*}}+1>0
$$

Furthermore, the inequality (35a) along with the equation (37) yields the relation $2 \sqrt{\varepsilon}<\bar{\mu}_{*}^{2 \sigma}$. This result together with the ones given in (39) and (40) point to the correctness of the inequality (38) (note that $\alpha_{*}>\alpha_{*}$ and $\mathbf{x}_{*} \mathbf{x}_{*}^{T}<R$ ). This is an indication of the fact that the function $\bar{f}(\bar{x}, \mu)+\varepsilon-$ $\left(\alpha_{*}+\sqrt{\varepsilon}\right)\left(\mu^{2 \sigma}+\varepsilon^{2}\right)$ is negative at the point $\left(\overline{\mathbf{x}}_{*}, \bar{\mu}_{*}\right)$ lying on the unit sphere. Thus, it can be inferred from Theorem 1 that $\alpha_{\varepsilon}$ is less than $\alpha_{*}+\sqrt{\varepsilon}$.

For the case when $f(\mathbf{x})$ attains its infimum, Theorem 5 presents an open interval ending at the origin such that for any $\varepsilon$ in this interval, the error between $\alpha_{*}$ and $\alpha_{\varepsilon}$ is guaranteed not to exceed $\sqrt{\varepsilon}$. However, this bound is useful only when it is initially known that $f(\mathbf{x})$ attains its infimum. It is worth mentioning that although the assumption that $f(\mathbf{x})$ attains its infimum has been made in several papers such as [24], its verification can be a formidable task. Nonetheless, there exist some works in the literature proposing sufficient conditions for a polynomial to attain its infimum; e.g., see [30].

Remark 3: Let the results of Theorems 2, 3, 4 and 5 be encapsulated here. An open interval with one end at the origin can be obtained such that $\alpha_{\varepsilon}$ is an upper bound on $\alpha_{*}$ for any $\varepsilon$ in this interval. Furthermore, if this interval is replaced by a tighter one, the function $\alpha_{\varepsilon}$ will be monotone increasing over it, which implies that smaller $\varepsilon$ in this interval results in an $\alpha_{\varepsilon}$ closer to $\alpha_{*}$. Besides, as $\varepsilon$ goes to zero, the function $\alpha_{\varepsilon}$ converges to $\alpha_{*}$. Finally, in the case when the infimum $\alpha_{*}$ is attainable, if certain a priori information is available, one can simply find a number $\varepsilon$ for which a desirable level of accuracy in estimating $\alpha_{*}$ with $\alpha_{\varepsilon}$ is guaranteed.

\section{THE INFIMUM OF A RATIONAL FUNCTION}

It is desired now to find the infimum of a rational function $\frac{f(\mathbf{x})}{h(\mathbf{x})}$, where $f(\mathbf{x})$ and $h(\mathbf{x})$ are coprime polynomials (these polynomials can be obtained by cancelling out the greatest common divisor of the numerator and denominator of the rational function, if necessary, using the available methods; e.g. see [31], [32], [33]). Suppose that $h(\mathbf{x})$ is not constant, because otherwise $\frac{f(\mathbf{x})}{h(\mathbf{x})}$ will be a polynomial and the method proposed earlier can be used accordingly to obtain its infimum. The following lemma is borrowed from [21].

Lemma 2: If $h(\mathbf{x})$ changes sign, then the infimum of $\frac{f(\mathbf{x})}{h(\mathbf{x})}$ is $-\infty$.

At this point, it is required to check whether or not $h(\mathbf{x})$ changes sign. This can sometimes be inferred from the nature of the polynomial $h(\mathbf{x})$. For example, when $h(\mathbf{x})$ is 
the square of another function, it is always nonnegative. In general, the technique introduced in the previous section can be employed to find the infimum of $h(\mathbf{x})$, leading to one of the following possibilities:

- The infimum of $h(\mathbf{x})$ is nonnegative: In this case, the polynomial $h(\mathbf{x})$ does not change sign.

- The infimum of $h(\mathbf{x})$ is negative and finite: In this case, the infimum of $\frac{f(\mathbf{x})}{h(\mathbf{x})}$ is $-\infty$ (note that if the infimum of a non-constant polynomial is finite, then its supremum is $+\infty)$.

- The infimum of $h(\mathbf{x})$ is $-\infty$ : Compute now the infimum of $-h(\mathbf{x})$. If the result is a negative value (finite or infinite), it means that $h(\mathbf{x})$ takes both negative and positive values, which implies that the infimum of $\frac{f(\mathbf{x})}{h(\mathbf{x})}$ is $-\infty$. Otherwise, $h(\mathbf{x})$ is always negative. In this case, negate both the numerator and the denominator of $\frac{f(\mathbf{x})}{h(\mathbf{x})}$ in order to make its denominator nonnegative for all $\mathbf{x} \in \Re^{n}$.

Without loss of generality, assume that $h(\mathbf{x})$ is always nonnegative. Denote the infimum of the function $\frac{f(\mathbf{x})}{h(\mathbf{x})}$ (to be obtained) with $\alpha_{*}$. Consider now a slack variable $\mu$ and rewrite the rational function $\frac{f\left(\frac{\mathbf{x}}{\mu}\right)}{h\left(\frac{\mathbf{x}}{\mu}\right)}$ as $\frac{\bar{f}(\mathbf{x}, \mu)}{h(\mathbf{x}, \mu)}$, where $\bar{f}(\mathbf{x}, \mu)$ and $\bar{h}(\mathbf{x}, \mu)$ are two homogeneous polynomials.

Definition 2: For any nonnegative integer $k$ and any $\varepsilon \geq$ 0 , let $\alpha_{\varepsilon}(k)$ denote the supremum of all values of $\alpha$ for which there exist polynomials $\phi_{1}^{\varepsilon}(\mathbf{x}, \mu) \in \Re_{k}[\mathbf{X}]$ and $\phi_{2}^{\varepsilon}(\mathbf{x}, \mu) \in$ $\Sigma^{2}$ such that:

$$
\begin{aligned}
\bar{f}(\mathbf{x}, \mu)+\varepsilon-\alpha\left(\bar{h}(\mathbf{x}, \mu)+\varepsilon^{2}\right) & =\left(1-\mathbf{x x}^{T}-\mu^{2}\right) \phi_{1}^{\varepsilon}(\mathbf{x}, \mu) \\
& +\phi_{2}^{\varepsilon}(\mathbf{x}, \mu)
\end{aligned}
$$

Define also:

$$
\alpha_{\varepsilon}:=\lim _{k \rightarrow+\infty} \alpha_{\varepsilon}(k)
$$

Remark 4: In the case when $f(\mathbf{x})$ and $h(\mathbf{x})$ are homogeneous polynomials of the same degree, one can consider the equation:

$$
f(\mathbf{x})+\varepsilon-\alpha\left(h(\mathbf{x})+\varepsilon^{2}\right)=\left(1-\mathbf{x x}^{T}\right) \phi_{1}^{\varepsilon}(\mathbf{x})+\phi_{2}^{\varepsilon}(\mathbf{x})
$$

instead of the one given in (41) to define $\alpha_{\varepsilon}(k)$.

Pursuing the techniques given in the previous section, the following theorem is provided for attaining the infimums of rational functions, as an extension of the results proposed for polynomials.

Theorem 6: Consider an arbitrary point $\mathbf{x}_{0}$ for which $h\left(\mathbf{x}_{0}\right)$ is nonzero. The following statements are true about the infimum $\alpha_{*}$ :

i) The quantity $\alpha_{0}$ is a lower bound on $\alpha_{*}$.

ii) For any $\varepsilon$ satisfying the relation:

$$
0<\varepsilon<\max \left(\frac{h\left(\mathbf{x}_{0}\right)}{f\left(\mathbf{x}_{0}\right)},-\operatorname{sgn}\left(\frac{h\left(\mathbf{x}_{0}\right)}{f\left(\mathbf{x}_{0}\right)}\right) \times \infty\right)
$$

the inequality $\alpha_{*}<\alpha_{\varepsilon}$ holds (note that if $f\left(\mathbf{x}_{0}\right)$ is zero, then the inequality (44) is identical to $0<\varepsilon<\infty$ ).

iii) The function $\alpha_{\varepsilon}$ is monotone increasing (with respect to $\varepsilon$ ) over the interval

$$
\begin{gathered}
\left(0,-\bar{f}\left(\overline{\mathbf{x}}_{0}, \bar{\mu}_{0}\right)+\sqrt{\bar{f}\left(\overline{\mathbf{x}}_{0}, \bar{\mu}_{0}\right)^{2}+\bar{h}\left(\overline{\mathbf{x}}_{0}, \bar{\mu}_{0}\right)}\right), \text { where: } \\
\bar{\mu}_{0}=\frac{1}{\sqrt{1+\mathbf{x}_{0} \mathbf{x}_{0}^{T}}}, \quad \overline{\mathbf{x}}_{0}=\bar{\mu}_{0} \times \mathbf{x}_{0}
\end{gathered}
$$

iv) The infimum $\alpha_{*}$ can be obtained from the following relation:

$$
\alpha_{*}=\lim _{\varepsilon \rightarrow 0^{+}} \alpha_{\varepsilon}
$$

v) Assume that the infimum of $f(\mathbf{x})$ is attainable with a minimizer $\mathbf{x}_{*}$. Let $R$ denote the radius of a ball centered at the origin in which $\mathbf{x}_{*}$ is located. Moreover, denote a lower bound on $\alpha_{*}$ with $\alpha_{*}$. For any $\varepsilon$ satisfying all the relations:

$$
\begin{aligned}
& 0<\varepsilon<\frac{\bar{h}\left(\overline{\mathbf{x}}_{*}, \bar{\mu}_{*}\right)^{2}}{4}, \\
& 0<\varepsilon<\max \left(\frac{h\left(\mathbf{x}_{0}\right)}{f\left(\mathbf{x}_{0}\right)},-\operatorname{sgn}\left(\frac{h\left(\mathbf{x}_{0}\right)}{f\left(\mathbf{x}_{0}\right)}\right) \times \infty\right), \\
& 0<\varepsilon<\max \left(\frac{1}{-\underline{\alpha_{*}}}, \operatorname{sgn}\left(\underline{\alpha_{*}}\right) \times \infty\right)
\end{aligned}
$$

the inequality given below holds:

$$
\alpha_{*}<\alpha_{\varepsilon}<\alpha_{*}+\sqrt{\varepsilon}
$$

where:

$$
\bar{\mu}_{*}=\frac{1}{\sqrt{1+\mathbf{x}_{*} \mathbf{x}_{*}^{T}}}, \quad \overline{\mathbf{x}}_{*}=\bar{\mu}_{*} \times \mathbf{x}_{*}
$$

(if $\alpha_{*}$ is equal to 0 , replace the inequality (47c) with $0<$ $\varepsilon<\infty)$.

Proof: The proofs of different parts of this theorem can be carried out in line with those of Theorems 2, 3, 4 and 5, and Corollary 1 outlined for the case of polynomials (note that in the case of polynomials, $\mathbf{x}_{0}$ was assumed to be equal to 0 , and consequently $h\left(\mathbf{x}_{0}\right)=1$ ). The details are omitted here.

Remark 5: A lower bound $\alpha_{*}$ on the infimum $\alpha_{*}$ can be obtained using the existing methods in the literature. For instance, compute the supremum of all values of $\alpha$ for which $f(\mathbf{x})-\alpha h(\mathbf{x})$ is SOS. If this supremum value is finite, $\alpha_{*}$ can be set equal to it.

Remark 6: In order for part (v) of Theorem 6 to be usable, some a priori knowledge is required. First, it is required that the infimum $\alpha_{*}$ be attainable. Furthermore, the radius of a ball in which a minimizer of $\frac{f(\mathbf{x})}{h(\mathbf{x})}$ lies is required to be known. More importantly, it is necessary that a positive lower bound on $\bar{h}\left(\overline{\mathbf{x}}_{*}, \bar{\mu}_{*}\right)$ be computed (in order to find a subinterval of the region specified by (47a)). This can be simple or quite challenging depending on the nature of the given rational function.

\section{NUMERICAL EXAMPLES}

The following simulations are run using the software SOSTOOLS on a Dell computer with a Pentium IV $3.0 \mathrm{GHz}$ and $1 \mathrm{~GB}$ of RAM.

Example 1: Consider the polynomial $f(\mathbf{x})=x_{1}^{2} x_{2}^{2}\left(x_{1}^{2}+\right.$ $\left.x_{2}^{2}-1\right)$. The global minimum of this polynomial is equal to $\frac{1}{27}$, which occurs at $x_{1}^{2}=x_{2}^{2}=\frac{1}{3}$. The work [16] applies 
its proposed method to this example and arrives at the lower bound -33.1573 . Now, let the present method be employed to treat this problem. Since $f(0)$ is equal to 0 , the statements of Theorems 2, 3 and 4 can be expressed as follows:

- For any $\varepsilon$ belonging to the open interval $(0, \infty)$, the quantity $\alpha_{\varepsilon}$ is an upper bound on the infimum $\alpha_{*}$.

- Let $\alpha_{\varepsilon}$ be regarded as a function of $\varepsilon$. This function is monotone increasing over the interval $(0,1)$.

- As $\varepsilon$ goes to $0^{+}$, the term $\alpha_{\varepsilon}$ converges to the infimum $\alpha_{*}$.

For eight different fixed values of $\varepsilon$ in the region $(0,1]$, the term $\alpha_{\varepsilon}(k)$ is computed for $k$ equal to 4,6 and 8 (to find a proper estimate of $\alpha_{\varepsilon}$ ). The resultant errors $\alpha_{\varepsilon}(k)-\alpha_{*}$ for all cases are tabulated in Table I. This table demonstrates that unlike the result obtained by the method in [16], relatively precise results are attained by solving small-sized SOS problems using the present method. The corresponding computation times for $\alpha_{\varepsilon}(6)$ are shown in Table II to demonstrate the speed of the proposed SOS technique.

On the other hand, one can use Theorem 5 to obtain a solution with a prespecified level of accuracy. For this purpose, a lower bound on $\alpha_{*}$ (which was denoted by $\underline{\alpha_{*}}$ earlier) can be chosen as -33.1573 . This bound is, in fact, borrowed from [16], as noted above. One should take note of the fact that $\alpha_{*}$ is not positive, as $f(0)=0$ is equal to 0 . Moreover, $f(\mathbf{x})$ is positive whenever its factor $x_{1}^{2}+x_{2}^{2}-1$ is positive. This means that $f(\mathbf{x})$ attains its infimum with a minimizer located in the unit ball centered at the origin. Therefore, $R$ can be chosen as 1 . The inequalities (35a), (35b) and (35c) can hence be reduced to $0<\varepsilon<0.0039$, $0<\varepsilon<\infty$ and $0<\varepsilon<0.0302$, respectively. Thus, Theorem 5 states that for any $\varepsilon$ in the interval $(0,0.0039)$, the error $\alpha_{\varepsilon}-\alpha_{*}$ will be between 0 and $\sqrt{\varepsilon}$.

Example 2: It is desired to obtain the infimum of following polynomial:

$$
\begin{aligned}
f(\mathbf{x}) & =\left(x_{1}-1\right)^{4}\left(x_{2}-1\right)^{2}+\left(x_{1}-1\right)^{2}\left(x_{2}-1\right)^{4}+x_{3}^{6} \\
& -3\left(x_{1}-1\right)^{2}\left(x_{2}-1\right)^{2} x_{3}^{2}
\end{aligned}
$$

It follows from the well-known result on the Motzkin polynomial that $\alpha_{*}$ is equal to 0 in this case. In what follows, the efficacy of different approaches is tested on this example:

- The work [17] arrives at the lower bound $-\infty$, which is by no means useful.

- Let $k$ denote the maximum degrees of the polynomials in the equation (11) of the work [24]. By setting $k$ equal to 4,6 and 8 , one will attain the respective lower bounds $-0.16 \times 10^{-5},-0.23 \times 10^{-6}$ and $-0.23 \times 10^{-6}$, with the running times 5.1406, 9.8906 and 43.89 seconds, respectively.

- Using the current work, the quantity $\alpha_{\varepsilon}(k)$ is obtained as $0.28 \times 10^{-7}$ after $4.47 \mathrm{sec}$, for $k=8$ and $\varepsilon=10^{-8}$.

The above discussions demonstrate the superiority of the approach proposed in this paper over the existing ones. It is to be noted that the proposed method is more efficient in terms of both CPU time and the accuracy of the solution obtained.

Example 3: Consider the following rational function:

$$
\frac{f(\mathbf{x})}{h(\mathbf{x})}=\frac{x_{1}^{4} x_{2}^{2}+x_{2}^{4} x_{3}^{2}+x_{3}^{4} x_{1}^{2}-3 x_{1}^{2} x_{2}^{2} x_{3}^{2}}{\left(x_{1}^{2}+2 x_{1}^{2} x_{2}^{2}+x_{2}^{2}\right) x_{3}^{4}}
$$

It can be easily observed that the denominator of this function is SOS. Besides, its numerator is nonnegative in light of the arithmetic mean-geometric mean inequality. These two observations point to the fact that $\frac{f(\mathbf{x})}{h(\mathbf{x})}$ is always nonnegative. On the other hand, this function becomes zero for $x_{1}=$ $x_{2}=x_{3}$. As a result, the infimum of $\frac{f(\mathbf{x})}{h(\mathbf{x})}$ is equal to 0 . Now, let different approaches be exploited to compare their effectiveness in this example. If one commences from the SOS formula given in both of the works [27] and [21], SeDuMi runs into numerical problems and gives the number -7780.7 . This wrong result demonstrates that the methods introduced in [27] and [21] may diverge, even in simple problems. In contrast, $\alpha_{\varepsilon}(k)=0.68 \times 10^{-6}$ is obtained in $1.48 \mathrm{sec}$, for $\varepsilon=10^{-7}$ and $k=6$. This substantiates that a fairly precise solution is attained quickly in this example using the present work.

\section{Conclusions}

In this paper, the problem of computing the infimum of a rational function is investigated. This problem plays a key role in control design and performance analysis for many real-world systems. It is first shown that the infimum of a polynomial function can be obtained by solving a family of SDP problems. The result is then extended to the case of a rational function. Different aspects of the underlying ideas are exhaustively explored to show the merit of this work. Some numerical examples are ultimately given to clarify the proposed techniques and demonstrate their efficacies.

\section{REFERENCES}

[1] D. Henrion and J. B. Lasserre, "Solving nonconvex optimization problems - How GloptiPoly is applied to problems in robust and nonlinear control," IEEE Control Systems Magazine, vol. 24, no. 3, pp. 72-83, 2004.

[2] C. W. Scherer, "LMI relaxations in robust control," European Journal of Control, vol. 12, no. 1, pp. 3-29, 2006.

[3] R. C. L. F. Oliveira and P. L. D. Peres, "LMI conditions for robust stability analysis based on polynomially parameter-dependent Lyapunov functions," Systems \& Control Letters, vol. 55, no. 1, pp. 52-61, 2006.

[4] J. Lavaei and A. G. Aghdam, "A necessary and sufficient condition for robust stability of LTI discrete-time systems using sum-of-squares matrix polynomials," in Proceedings of 45th IEEE Conference on Decision and Control, San Diego, CA, pp. 2924-2930, 2006.

[5] D. Henrion and A. Garulli, Positive polynomials in control, Lecture Notes in Control and Information Sciences, Springer Verlag, Berlin, vol. 312, 2005.

[6] P. Dorato, "Quantified multivariate polynomial inequalities - The mathematics of practical control design problems," IEEE Control Systems Magazine, vol. 20, no. 5, pp. 48-58, 2000.

[7] J. Löfberg, "A toolbox for modeling and optimization in MATLAB," in Proceedings of the CACSD Conference, Taipei, Taiwan, 2004 (available online at http://control.ee.ethz.ch/ joloef/yalmip.php).

[8] S. Prajna, A. Papachristodoulou, P. Seiler and P. Parrilo, "SOSTOOLS sum of squares optimization toolbox for MATLAB," Users guide, 2004 (available online at http://www.cds.caltech.edu/sostools).

[9] P. Sjovall, T. McKelvey and T. Abrahamsson, "Constrained statespace system identification with application to structural dynamics," Automatica, vol. 42, no. 9, pp. 1539-1546, 2006. 
TABLE I

THE QUANTITY $\alpha_{\varepsilon}(k)-\alpha_{*}$ FOR EXAMPLE 1

\begin{tabular}{|l|l|l|l|l|l|l|l|l|}
\hline & $\varepsilon=1$ & $\varepsilon=10^{-1}$ & $\varepsilon=10^{-2}$ & $\varepsilon=10^{-3}$ & $\varepsilon=10^{-4}$ & $\varepsilon=10^{-5}$ & $\varepsilon=10^{-6}$ & $\varepsilon=10^{-7}$ \\
\hline$k=4$ & 0.5370 & 0.1360 & 0.0395 & -0.0200 & -0.1590 & -0.5453 & -0.6986 & -1.0151 \\
\hline$k=6$ & 0.5370 & 0.1360 & 0.0395 & 0.0046 & $0.46 \times 10^{-3}$ & $0.46 \times 10^{-4}$ & $0.50 \times 10^{-5}$ & $0.37 \times 10^{-7}$ \\
\hline$k=8$ & 0.5370 & 0.1360 & 0.0395 & 0.0046 & $0.46 \times 10^{-3}$ & $0.46 \times 10^{-4}$ & $0.50 \times 10^{-5}$ & $0.37 \times 10^{-7}$ \\
\hline
\end{tabular}

TABLE II

THE COMPUTATION TIME OF $\alpha_{\varepsilon}(k)$ FOR EXAMPLE 1

\begin{tabular}{|l|l|l|l|l|l|l|l|l|}
\hline & $\varepsilon=1$ & $\varepsilon=10^{-1}$ & $\varepsilon=10^{-2}$ & $\varepsilon=10^{-3}$ & $\varepsilon=10^{-4}$ & $\varepsilon=10^{-5}$ & $\varepsilon=10^{-6}$ & $\varepsilon=10^{-7}$ \\
\hline$\alpha_{\varepsilon}(6)$ & $0.47 \mathrm{sec}$ & $0.51 \mathrm{sec}$ & $0.66 \mathrm{sec}$ & $0.73 \mathrm{sec}$ & $0.97 \mathrm{sec}$ & $0.75 \mathrm{sec}$ & $1.4 \mathrm{sec}$ & $1.34 \mathrm{sec}$ \\
\hline
\end{tabular}

[10] L. X. Wang and F. Wan, "Structured neural networks for constrained model predictive control," Automatica, vol. 37, no. 8, pp. 1235-1243, 2001.

[11] I. A. Fotiou, P. Rostalski, P. A. Parrilo, and M. Morari, "Parametric optimization and optimal control using algebraic geometry methods," International Journal of Control, vol. 79, no. 11, pp. 1340-1358, 2006.

[12] D. Famularo, P. Pugliese and Y. D. Sergeyev, "A global optimization technique for checking parametric robustness," Automatica, vol. 35, no. 9 , pp. $1605-1611,1999$.

[13] S. Hui, W. E. Lillo and S. H. Zak, "Solving minimum norm problems using penalty functions and the gradient method," Automatica, vol. 31, no. 1, pp. 115-124, 1995.

[14] D. Jibetean and B. Hanzon, "Linear matrix inequalities for global optimization of rational functions and $\mathrm{H}_{2}$ optimal model reduction," in Proceedings of the Fifteenth International Symposium on Mathematical Theory of Networks and Systems, 2002.

[15] N. Z. Shor, "Class of global minimum bounds of polynomial functions," Cybernetics and Systems Analysis, vol. 23, no. 6, pp. 731-734, 1987.

[16] J. B. Lasserre, "Global optimization with polynomials and the problem of moments," SIAM Journal on Optimization, vol. 11, no. 3, pp. 796817, 2001.

[17] P. Parrilo and B. Sturmfels, "Minimizing polynomial functions," Discrete Mathematics and Theoretical Computer Science, vol. 60, pp. 83-99, 2003.

[18] G. Blekherman, "There are significantly more nonnegative polynomials than sums of squares," to appear in Israel Journal of Mathematics.

[19] G. Cassier, "Problème des moments sur un compact de $\Re^{n}$ et décomposition de polynômes à plusieurs variables," Journal of Functional Analysis, vol. 58, no. 3, pp. 254-266, 1984.

[20] D. Jibetean and M. Laurent, "Semidefinite approximations for global unconstrained polynomial optimization," SIAM Journal on Optimization, vol. 16, no. 2, pp. 490-514, 2005.

[21] D. Jibetean and E. D. Klerk, "Global optimization of rational functions: a semidefinite programming approach," Mathematical Programming, vol. 106, no. 1, pp. 93-109, 2006.

[22] J. B. Lasserre, "A sum of squares approximation of nonnegative polynomials," SIAM Journal on Optimization, vol. 16, no. 3, pp. 751765, 2006

[23] B. Hanzon and D. Jibetean, "Global minimization of a multivariate polynomial using matrix methods," Journal of Global Optimization, vol. 27, no. 1, pp. 1-23, 2003.

[24] J. Nie, J. Demmel and B. Sturmfels, "Minimizing polynomials via sum of squares over the gradient ideal," Mathematical Programming, vol. 106, no. 3, pp. 587-606, 2006.

[25] M. Schweighofer, "Global optimization of polynomials using gradient tentacles and sums of squares," to appear in SIAM Journal on Optimization.

[26] M. Putinar, "Positive polynomials on compact semi-algebraic sets," Indiana University Mathematics Journal, vol. 42, no. 3, pp. 969-984, 1993.

[27] J. Nie, J. Demmel and M. Gu, "Global minimization of rational functions and the nearest GCDs," to appear in Journal of Global Optimization.

[28] J. Nie and M. Schweighofer, "On the complexity of Putinar's Positivstellensatz," Journal of Complexity, vol. 23 , no. 1, pp. 135-150, 2007.
[29] E. de Klerk, M. Laurent and P. A. Parrilo, "On the equivalence of algebraic approaches to the minimization of forms on the simplex," Positive Polynomials in Control, Lecture Notes in Control and Information Sciences, Springer, vol. 312, pp. 121-132, 2005.

[30] M. Marshall, "Optimization of polynomial functions," Canadian Mathematical Bulletin, vol. 47, no. 4, pp. 575-587, 2003.

[31] K. Li, L. Zhi and M. T. Noda, "Solving approximate GCD of multivariate polynomials by Maple/Matlab/C combination," Proceedings of the Asian Technology Conference in Mathematics, pp. 492-499, Chiangmai, Thailand, 2000.

[32] L. Zhi and M. T. Noda, "Approximate GCD of multivariate polynomials " in Proceedings of the Asian Symposium on Computer Mathematics, pp. 9-18, Chiangmai, Thailand, 2000.

[33] M. A. Ochi, M. T. Noda and T. Sasaki, "Approximate greatest common divisor of multivariate polynomials and its application to illconditioned systems of algebraic equations," Journal of Information Processing, vol. 14, no. 3, pp. 292-300, 1991. 